\title{
Mitochondrial DNA haplogroups and ageing mechanisms in osteoarthritis
}

\author{
Ana M Valdes, ${ }^{1,2}$ Mary B Goldring ${ }^{3,4}$
}

\section{MITOCHONDRIAL DNA AND AGEING}

Osteoarthritis (OA) is the most common form of arthritis affecting more than 12\% of people over the age of $60 .^{1}$ Although late-onset articular cartilage degeneration is common and age is one of the most important risk factors for the disease, the relationship between old age and $\mathrm{OA}$ is not fully understood. ${ }^{2}$ In the past it was believed that the link with age was due to 'wear and tear' of articular cartilage by continuous mechanical stress; we now know, however, that OA involves an active response to injury comprising remodelling of articular cartilage and subchondral bone, in addition to synovial inflammation and damage to other joint structures such as ligaments and menisci. ${ }^{3}$

Biological ageing is a complex process and it is now widely accepted that ageing starts with molecular damage, leading to cell, tissue and, ultimately, organ dysfunction. $^{4}$ Extensive evidence from animal models and in vitro studies indicates that mitochondria contribute to specific aspects of the ageing process, including cellular senescence, chronic inflammation and the age-dependent decline in stem cell activity. ${ }^{5}$

Perhaps the best known and most longstanding hypothesis to explain ageing is the free radical theory that proposes a central role for the mitochondrion as the principal source of intracellular reactive oxygen species (ROS) leading to mitochondrial DNA (mtDNA) mutations. ${ }^{4} 5$ Somatic (acquired) mtDNA mutations and their association with the decline in mitochondrial function during ageing are well described, but these observations do not necessarily imply a causal relationship between mitochondrial dysfunction and human ageing. The maternally inherited mtDNA sequences encode the key proteins involved in energy production, although

\footnotetext{
${ }^{1}$ School of Medicine, University of Nottingham, Nottingham, UK; ${ }^{2}$ Arthritis Research UK Pain Centre, University of Nottingham, Nottingham, UK; ${ }^{3}$ Hospital for Special Surgery, HSS Research Institute, New York, New York, USA; ${ }^{4}$ Department of Cell and Developmental Biology, Weill Cornell Medical College, New York, New York, USA

Correspondence to Dr Ana M Valdes, Academic Rheumatology, Clinical Sciences Building, Nottingham City Hospital Hucknall Road, Nottingham NG5 1PB, UK; ana.valdes@nottingham.ac.uk
}

the relevance of high sequence variability of mtDNA had been considered of little functional relevance. Latorre-Pellicer and coauthors showed recently that transferring mtDNA from a mouse strain to the nuclear DNA (nDNA) background of another strain results in huge differences in insulin signalling, obesity and longevity throughout the life of the mouse. ${ }^{6}$ The two mtDNA sequences differ in genetic variants that confer 12 amino acid substitutions and 12 changes in RNA molecules involved in mitochondrial protein synthesis; this level of variation is enough to result in striking differences in the ROS generation, insulin signalling, obesity and cell-senescence-related parameters such as telomere shortening and mitochondrial dysfunction. Showing the direct relevance of mtDNA in human ageing and in age-related diseases, such as OA, is a big challenge and one which is, at least in part, addressed in this issue. ${ }^{7}$

\section{mtDNA in OA}

Over the past 10 years, the group led by Francisco Blanco and Ignacio Rego-Perez has shown that differences in mtDNA haplogroups correspond to variations in the prevalence and progression of cartilage loss in large joint $\mathrm{OA}^{8}$ In a series of studies from Spanish OA cases and controls, the evidence has accumulated for an association between $\mathrm{OA}$ prevalence and the J haplogroup ${ }^{10}$ (table 1). However, two studies in samples from the UK have failed to find an association with the J haplotype, ${ }^{9-11}$ whereas evidence of association of the $\mathrm{T}$ haplotype with lower disease risk was found in a small UK cohort $^{9}$ (table 1).

The mtDNA haplogroups $\mathrm{J}$ and $\mathrm{T}$ share the same phylogenetic origin and a set of common uncoupling mitochondrial polymorphisms. ${ }^{12}$ These uncoupling polymorphisms confer different metabolic characteristics compared with other mitochondrial lineages, particularly the most common and highly efficient mtDNA haplogroup H. ${ }^{13}$

The jury is still out regarding the role of mtDNA $\mathrm{T}$ and $\mathrm{J}$ haplogroups with regard to genetic susceptibility in populations with large joint $\mathrm{OA}$, particularly when compared with the evidence accumulated for nuclear genetic variants identified from genome wide association studies (GWAS) or otherwise. ${ }^{14}$ To date, eight variants associated with knee OA have been reported with significance of $\mathrm{p}<1 \times 10^{-7}$ and 11 variants with hip OA in Caucasians. At least three other variants have been reported at high significance levels in Asians (see ref. 14 for details).

On the other hand, with the exception of variants mapping to GDF5 and FTO genes, the mechanisms underlying the risk conferred by variants linked to knee OA are yet to be unveiled. ${ }^{14}$ Importantly, as of today, very few efforts have been made to identify genetic risk factors contributing to risk of progression or incidence of disease.

The mtDNA haplotypes T, J and the JT cluster, on the other hand, are significantly associated in populations from the USA, the Netherlands and Spain with radiographic incidence and progression of the disease ${ }^{7} 15$ (table 1). FernandezMoreno and coauthors report that the mtDNA haplogroup $J$, the same haplogroup associated with lower OA prevalence, lower disease progression and lower cartilage loss, is also associated with a significantly lower risk of incident knee $\mathrm{OA}$ in a population of 3124 individuals from two prospective cohorts from the Netherlands and the USA. ${ }^{7}$

\section{FUNCTIONAL ANALYSIS OF MTDNA VARIANTS}

From previous studies, it is known that the low OA risk haplogroup $\mathrm{J}$ is associated with lower serum levels of markers of collagen type-II degradation and of matrix metalloproteinases, but all of these studies failed to address the key question arising from this large body of evidence: 'What is the functional role of these mtDNA haplogroups?'

To answer this question, FernandezMoreno et $\mathrm{al}^{7}$ used cytoplasmic hybrid (cybrid) cell lines. Cybrids incorporate mitochondria from human subjects and perpetuate the mtDNA-encoded components while maintaining the nuclear background of different cybrid lines as constant. ${ }^{16}$ Thus, this technique allows investigators to assess the influence of mtDNA variation on cell function. To investigate the role of mtDNA haplogroups, they also created cybrids using osteosarcoma cell lines with the same nuclear background, one of them harbouring the haplogroup $\mathrm{J}$ (which protects against $\mathrm{OA}$ ) and another harbouring the haplogroup $\mathrm{H}$ (linked to higher risk of $\mathrm{OA})$.

The cybrids carrying the haplogroup $\mathrm{H}$ produced higher ATP levels than those 
Table 1 Selected associations between T, J and TJ cluster mitochondrial DNA and OA prevalence, progression and incidence of OA

\begin{tabular}{|c|c|c|c|c|c|c|}
\hline Study & Origin & Haplogroup & Trait studied & Total N & $\begin{array}{l}\text { Effect }(95 \% \mathrm{Cl}) \\
p \text { value }\end{array}$ & Reference \\
\hline Case-control & Spain & $\mathrm{J}$ & OA prevalence & 2557 OA, 1339 controls & $\mathrm{OR}=0.57(0.46$ to 0.71$) \mathrm{p}<0.00001$ & 10 \\
\hline Case-control & UK & $J$ & OA prevalence & 7846 OA, 5402 controls & $\mathrm{OR}=1.19(0.72$ to 1.95$) \mathrm{ns}$ & 10 \\
\hline Case-control & UK & $\mathrm{T}$ & $\mathrm{OA}$ prevalence & 453 OA, 280 controls & $\mathrm{OR}=0.57(0.35$ to $0.940 \mathrm{p}<0.027$ & 9 \\
\hline CHECK cohort & The Netherlands & $\mathrm{T}$ & OA progression & $431 \mathrm{OA}$ & $\mathrm{HR}=0.645(0.419$ to 0.978$) \mathrm{p}<0.05$ & 15 \\
\hline OAI & USA & $\mathrm{T}$ & OA progression & 891 OA & $\mathrm{HR}=0.50(0.28$ to 0.88$) \mathrm{p}<0.05$ & 24 \\
\hline Spanish OA cohort & Spain & $\mathrm{T}$ & OA progression & $281 \mathrm{OA}$ & $\mathrm{HR}=0.69(0.38$ to 1.28$) \mathrm{ns}$ & 25 \\
\hline Meta analysis & & $\mathrm{T}$ & $\mathrm{OA}$ progression & $1603 \mathrm{OA}$ & $H R=0.61(0.45$ to 0.82$) p=0.001$ & 15 \\
\hline CHECK cohort & The Netherlands & $\mathrm{JT}$ & OA progression & $431 \mathrm{OA}$ & $\mathrm{HR}=0.71(0.50$ to 0.96$) \mathrm{p}<0.05$ & 15 \\
\hline OAI & USA & JT & OA progression & 891 OA & $\mathrm{HR}=0.81(0.59$ to 1.11$) \mathrm{ns}$. & 15 \\
\hline Spanish OA cohort & Spain & $\mathrm{JT}$ & OA progression & $281 \mathrm{OA}$ & $\mathrm{HR}=0.80(0.50$ to 1.26$) \mathrm{ns}$ & 15 \\
\hline Meta analysis & & JT & & $1603 \mathrm{OA}$ & $H R=0.77(0.62$ to 0.94$) p=0.009$ & 15 \\
\hline CHECK cohort & The Netherlands & $\mathrm{J}$ & OA incidence & 635 & $\mathrm{HR}=0.73(0.47$ to 1.00$) \mathrm{p}<0.05$ & 7 \\
\hline OAI & USA & $J$ & OA incidence & 2579 & $H R=0.68(0.47$ to 0.97$) p<0.05$ & 7 \\
\hline Meta analysis & & $J$ & OA incidence & 3214 & $\mathrm{HR}=0.70(0.54$ to 0.91$) \mathrm{p}=0.006$ & 7 \\
\hline
\end{tabular}

OA, osteoarthritis; OAl, osteoarthritis initiative.

with the haplogroup J, but this higher energetic efficiency was accompanied by higher production of ROS and the proportion of cells that survived in the presence of hydrogen peroxide was almost half the number of cybrids with haplogroup J. In chondrocytes during OA, oxidative stress may act together with inflammatory and/or mechanical stress to accentuate catabolic processes by increasing the levels of ROS relative to antioxidants. ${ }^{17} 18$ The increased levels of ROS also contribute to the senescence secretory phenotype, in which the age-related decline in the responses of chondrocytes to anabolic growth factors are related to increased oxidative stress. ${ }^{19} 20$ The depletion of antioxidants promotes mitochondrial dysfunction in chondrocytes, ${ }^{21}$ which in turn can amplify the stress responses through increased production of nitric oxide and ROS and activation of $\mathrm{NF}-\kappa \mathrm{B}$ signalling. ${ }^{21-23}$

In the presence of staurosporine, which induces cell apoptosis, the cybrids with the haplogroup $\mathrm{H}$ had over 50\% more apoptotic cells than the cybrids with the low OA risk haplogroup $\mathrm{J}^{7}$ These data, therefore, prove the functional relevance of mtDNA variation linked to risk of OA on cell function and survival and is in agreement with recent work by the same group showing that OA cartilage exhibits signs of early molecular ageing compared with healthy age-matched cartilage. ${ }^{2}$

\section{CLINICAL RELEVANCE}

The data accumulated on the role of mtDNA on cell function and on OA risk have potential clinical implications. On the one hand, it may allow investigators in the future to define an 'age-related OA' genetic type (haplogroup $\mathrm{H}$ ) versus one which is protected from the effects of ageing. This group with lower incidence and progression can be excluded from clinical studies that require rapidly progressing $\mathrm{OA}$ populations. At the same time, haplogroup $\mathrm{J}$ carriers are not fully protected from OA; therefore, studying risk factors in this haplogroup can help identify a group of individuals where other molecular mechanisms linked to OA, for example, those derived from bone changes or from inflammation, may be stronger predictors for progression. These data also raise the important question of the contribution of interactions between nDNA and mtDNA haplogroups, which have yet to be investigated. Finally, $\mathrm{OA}$ is a disease that occurs together with cardiometabolic comorbidities which are known to be influenced by mitochondrial dysfunction. Haplogroup $\mathrm{H}$ carriers may therefore be the group of OA sufferers at higher risk of metabolic syndrome and cardiovascular disease and with the most chance to benefit from regenerative therapies targeting early cartilage damage or, at more advanced stages, early joint replacement.

Funding Research related to this topic was supported by Arthritis Research UK Pain Centre grant \#18769 (AMV) and National Institutes of Health grants R01-AG022021 and RC4-AR060546 (MBG).

Competing interests None declared.

Provenance and peer review Commissioned; externally peer reviewed.

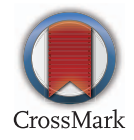

To cite Valdes AM, Goldring MB. Ann Rheum Dis 2017;76:939-941.

Received 30 November 2016

Accepted 17 December 2016

Published Online First 9 January 2017

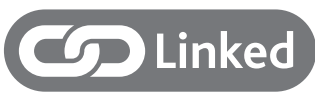

- http://dx.doi.org/10.1136/annrheumdis-2016210131

Ann Rheum Dis 2017;76:939-941.

doi:10.1136/annrheumdis-2016-210783

\section{REFERENCES}

1 Zhang Y, Jordan JM. Epidemiology of osteoarthritis. Clin Geriatr Med 2010;26:355-69.

2 Vidal-Bralo L, Lopez-Golan Y, Mera-Varela A, et al. Specific premature epigenetic aging of cartilage in osteoarthritis. Aging (Albany NY) 2016:8:2222-31.

3 Goldring MB. Chondrogenesis, chondrocyte differentiation, and articular cartilage metabolism in health and osteoarthritis. Ther Adv Musculoskelet Dis 2012:4:269-85.

4 Valdes AM, Glass D, Spector TD. Omics technologies and the study of human ageing. Nat Rev Genet 2013:14:601-7.

5 Sun N, Youle RJ, Finkel T. The Mitochondrial Basis of Aging. Mol Cell 2016;61:654-66.

6 Latorre-Pellicer A, Moreno-Loshuertos R, Lechuga-Vieco AV, et al. Mitochondrial and nuclear DNA matching shapes metabolism and healthy ageing. Nature 2016;535:561-5.

7 Fernandez-Moreno M, Siti-Hermida A, Vazquez-Mosquera ME, et al. Mitochondrial DNA haplogroups influence the risk of incident knee osteoarthritis in OAI and CHECK cohorts. A meta-analysis and functional study. Ann Rheum Dis 2017;76:992-97.

8 Blanco FJ, Rego I, Ruiz-Romero C. The role of mitochondria in osteoarthritis. Nat Rev Rheumatol 2011;7:161-9.

9 Soto-Hermida A, Fernández-Moreno M, Oreiro N, et al. mtDNA haplogroups and osteoarthritis in different geographic populations. Mitochondrion 2014;15:18-23. 
10 Shen JM, Feng L, Feng C. Role of mtDNA haplogroups in the prevalence of osteoarthritis in different geographic populations: a meta-analysis. PLOS ONE 2014;9:e108896.

11 Hudson G, Panoutsopoulou K, Wilson I, et al. No evidence of an association between mitochondrial DNA variants and osteoarthritis in 7393 cases and 5122 controls. Ann Rheum Dis 2013:72:136-9.

12 Mishmar D, Ruiz-Pesini E, Golik P, et al. Natural selection shaped regional mtDNA variation in humans. Proc Natl Acad Sci USA 2003;100:171-6.

13 Kenney MC, Chwa M, Atilano SR, et al. Molecular and bioenergetic differences between cells with African versus European inherited mitochondrial DNA haplogroups: implications for population susceptibility to diseases. Biochim Biophys Acta 2014;1842:208-19.

14 Warner SC, Valdes AM. Genetic association studies in osteoarthritis: is it fairytale? Curr Opin Rheumatol 2017;29:103-9.

15 Fernandez-Moreno M, Soto-Hermida A, Vazquez-Mosquera ME, et al. A replication study and meta-analysis of mitochondrial DNA variants in the radiographic progression of knee osteoarthritis. Rheumatology 2016. doi: 10.1093/rheumatology/ kew394. [Epub ahead of print 17 Nov 2016].

16 Vithayathil SA, Ma Y, Kaipparettu BA. Transmitochondrial cybrids: tools for functional studies of mutant mitochondria. Methods Mol Biol 2012;837:219-30.

17 Goldring MB, Berenbaum F. Emerging targets in osteoarthritis therapy. Curr Opin Pharmacol 2015;22:51-63.

18 Portal-Núñez S, Esbrit P, Alcaraz MJ, et al. Oxidative stress, autophagy, epigenetic changes and regulation by miRNAs as potential therapeutic targets in osteoarthritis. Biochem Pharmacol 2016;108:1-10.

19 Loeser RF. Integrins and chondrocyte-matrix interactions in articular cartilage. Matrix Biol 2014;39:11-16.

20 Loeser RF, Gandhi U, Long DL, et al. Aging and oxidative stress reduce the response of human articular chondrocytes to insulin-like growth factor 1 and osteogenic protein 1. Arthr Rheum 2014;66:2201-9.
21 Gavrilidis C, Miwa S, von Zglinicki T, et al. Mitochondrial dysfunction in osteoarthritis is associated with down-regulation of superoxide dismutase 2. Arthr Rheum 2013:65:378-87.

22 Vaamonde-García C, Riveiro-Naveira RR, Valcárcel-Ares MN, et al. Mitochondrial dysfunction increases inflammatory responsiveness to cytokines in normal human chondrocytes. Arthr Rheum 2012;64:2927-36

23 de Andrés MC, Maneiro E, Martín MA, et al. Nitric oxide compounds have different effects profiles on human articular chondrocyte metabolism. Arthritis Res Therapy 2013;15:R115.

24 Soto-Hermida A, Fernández-Moreno M, Oreiro N, et al. Mitochondrial DNA (mtDNA) haplogroups influence the progression of knee osteoarthritis. Data from the Osteoarthritis Initiative (OAI). PLOS ONE 2014;9:e112735.

25 Soto-Hermida A, Fernández-Moreno M, Pértega-Díaz $\mathrm{S}$, et al. Mitochondrial DNA haplogroups modulate the radiographic progression of Spanish patients with osteoarthritis. Rheumatol Int 2015;35:337-44. 